Historic, Archive Document

Do not assume content reflects current scientific knowledge, policies, or practices. 

Wholesale Catalogue

J. S. Department of Agriculture.

FOR THE

FALL AND SPRING OF 1893-4,

OF

\section{The Yokohama Gardeners' Association}

IMPORTERS AND GROWERS

OF

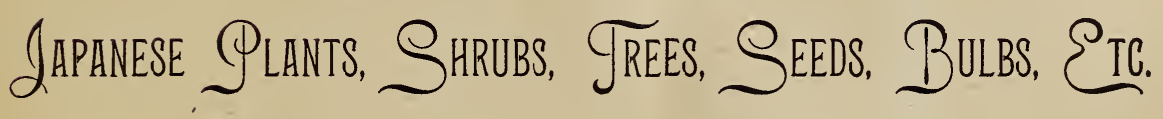

Glein Avenue, Near Piedmont Avenue,

OAKLAND, OAL.

Post Office Box 453, OAKLand, Cal.

Principal, Store and GaRden,

Nos. 21 to 35, Nakamura, Yokohama, Japan.

$\overline{\overline{\underline{2}}}$

CARRUTH \& CARRUTH, PRINTERS, OAKLAND.

1892. 



\title{
(0) REMARIXS.-(0)
}

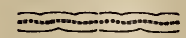

In presenting our catalogue for next fall, we desire to return our sincere thanks for the patronage bestowed on us by our many customers in the United States during the past season, and trust that they will continue to favor us with liberal orders for the coming season. We also solicit a trial from those who have not yet given us an order, and will try to merit their patronage and support, by using every endeavor in our power to comply with all their demands in a prompt and satisfactory manner.

The rapid progress in our business has enabled us to increase our stock, so that we now carry a large and beautiful variety of foliage, flowering and decorative plants, including very old and rare dwarf trees (all in fancy pots.) Customers and their friends will find many curiosities on visiting our nursery and garden.

As we have full descriptive catalogues of our principal store at Yokohama, Japan, we shall be very glad to furnish them to those who desire to import direct from Japan.

All orders should be accompanied by cash, either in the form of Draft, Check, Postoffice Order or Registered Letter, excepting from such as are well known to us.

We furnish all the goods, warranted first-class in every respect, and pack in a manner to insure safe arrival; but our responsibility ceases with delivery of goods to forwarders, be it Steamer, Railroad, Mail or Express.

Complaints of whatever nature must be made within a week after receipt of goods-otherwise they will not be taken cognizance of.

We take the utmost care to furnish the best seeds, but we give no warranty. Climate, soil and the cultivation of the same demands this proviso.

No charge is made for boxes or packing, nor for delivery to Freight Depots or Express Offices in Oakland or San Francisco.

Y'OKOHAMA GARDENERS' ASSOCIATION, Glen Avenue (near Piedmont Ave.),

P. O. Box 453, Oakland.

\author{
OAKLAND, CaL.
}





\section{-PRICE LIST.}

\section{Japanese Bulbs.}

These bulbs are packed in Japan with great care by experienced men in our own nursery, and can be relied upon.

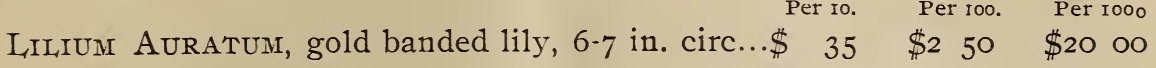

" " "

" "

" "

" " " " " "II I $/ 2$-I3 " extra I $00 \quad 8$ OO 5500

Each. Per тo. Per roo. Per 1000 .

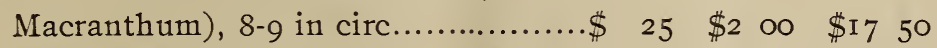

“ Auratum Platiphyllum (L. A.

Macranthum), 9-I 2 in circ.............. $50 \quad 4 \quad 50 \quad 2500$

“ Auratum Pictum, crimson-tipped at the ends of yellow rays................

"Auratum Rubro-Vittatum, petals are studded with purplish crimson spots with broad purplish crimson bands.....

"Virginale (L. Wittei), white with yellow bands without spots..............

"Virginal Albuir, white with yellow bands..............................

" Batemanni, orąnge yellow

" Concolor, lovely carmine studded... with black spots

" Concolor, var. Ohime, larger petals, deeper color than the preceding ........

" Coridion, lovely yellow. " " pure yellow without spots new variety

"CORDIFolium, heart-form leaves, white spreading flowers..................

“ ElEEGAnS, extra red.. ..................

" " A̧LICE WILSON, canary yellow

$45 \quad 350 \quad 2200$

$75 \quad 5 \quad 50 \quad 5000$

$75 \quad 6 \quad 50 \quad 55$ o०

50 $350 \quad 22$ о०

$55 \quad 450 \quad 40 \quad 00$

$35 \quad 2 \quad 50 \quad 2000$

I $00 \quad 800$

$35 \quad 3$ O० 2000

$50 \quad 350$

I 25 IO 00

$50 \quad 400$

$65 \quad 6$ oo 
JAPANESE BULBS-Continued.

LiLiUm Eliegans ORANGe, Sawa-yuri.........

" “ Atrosanguineum, . dark blood-red.

“ Elegans VARIEGATED, red and yellow variegated

“ Elemans Semipleno, small fringed erecting petals in center of flower, giving semi-double appearance which tipped with white.

“ El agans Flore Pleno, double flower

“ " INCOMPARABILE, red large flower..................................

“ BROWNI Or JAPONICA ODORUM, light cream within, purple outside the petals " BROWNI or JAPONICA OdORUM, large..

" LEICHTLINI, canary yellow with spots

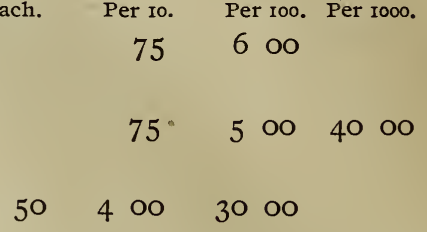

$500 \quad 4000$

550

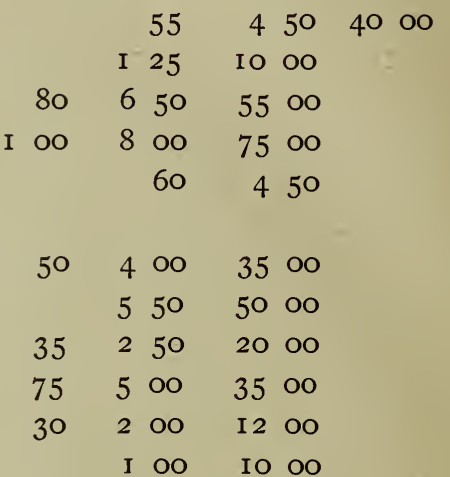

$35 \quad 3$ o0 2500

$60 \quad 450 \quad 35$ o0

$75 \quad 5$ o० 45 ००

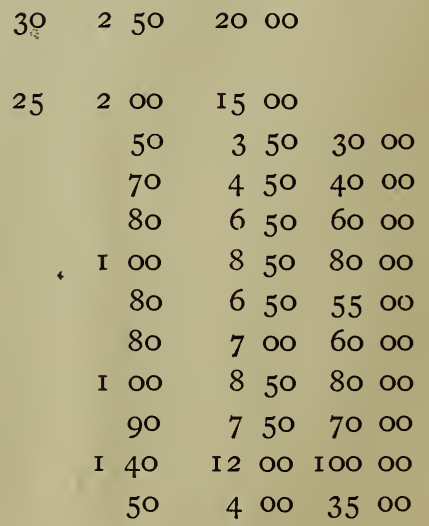


JAPANESE BUI.BS - Coutinued.

Lilium Tigrinum Pleno, double tiger lily...

" UKEYURI, white erecting flower.

Each. Per ro Per 100. Per rooo.

“ CALLOSUM,small lovely hanging flower

FRITILLARIA KAMTSCHATENSIS, small black

lily bell flower

JAPONICA, hanging yellow flower

with green veins spotted inside.........

NERINE JAPONICA, fire-red flowers, 2-3 in. circ

I 00750

$30 \quad 2$ OO I7 50

I $50, \quad$ I 2 OO

I $00 \quad 750$

$30 \quad 200 \quad$ I5 00

NERINE SARNIENSIS, pure yellow flowers.....

ERYTHRONIUM DENIS-CANIS, perennial of

lily family, small white flower.........

IxicoRIS RADIATA, yellowish red flowers.....

AMARYLLIS BELLADONA, reddish purple

flowers..................................

“ BELLADONA, deep rose shaded

with purple at the tips...................

ChINESE NARCissus, single \& double flowers

JAPANESE

" "

HeMEROCALIIS Flava, large Amaryllis-like yellow flowering herb

500

$\begin{aligned} \text { I } & \text { OO } \\ 50 & \end{aligned}$

I 50

I 25

$\begin{array}{lllll}75 & 6 & 50 & 60 & 00\end{array}$

$35 \quad 250 \quad 2000$ “

Flava, double flower.........

Crinum Astaticum two varieties..............

CALLA Aithiopica, 3-4 in. circ
“"
4-5
5-6
0.000000000
$\begin{array}{ll}6 & 6 \\ 6 & 6\end{array}$
6-7
400
6 oo
700

300

400

200

300

\section{Foliage, Flowering and Decorative Plants, Trees, Etc.}

A BIES FIRMA, 8-I2 in. pot grown

\section{Each.}

" BRACHYPHYLLA, rich foliage, valuable

timber tree pot grown, 8 -I 2 in.............. I oo 750

" BICOLOR, pot grown, 8-I2 in............... $75 \quad 500$

“ URAJIRO " " “

“ Томомі “ "

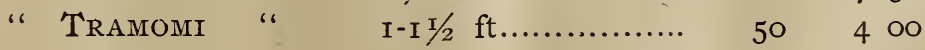

"VEITCHII " " 
Foliage, Flowering and Decorative Plants, Treis, Etc.-Con'd.

Acer Polymorphum, Japan Maples, from 3 to 5 varieties grafted on one stock, IO-I5 in. high

Eiach. Fer Io. Per 100.

Acer Polymorphum, two stocks twisted and grown together from 5 to 7 varieties.. ......

ACER Polymorphum, 40 varieties, I-I I $/ 2 \mathrm{ft} \ldots \ldots$.

$\begin{array}{llll}\text { " } & \text { " } & \mathrm{I} I / 2-2 \mathrm{I} / 2 \mathrm{ft} \ldots \ldots . \\ & \text { " } & 2 \mathrm{I} / 2-3 \mathrm{I} / 2 \mathrm{ft} \ldots \ldots .\end{array}$

Maple Seedlings, $\$ 4.00$ per roo. $\$ 30$ per Iоoo.

ACTINIA ARGUTA, climber, blooming in summer, small, sweet fruits, pot grown, I-I I $/ 2 \mathrm{ft} \ldots$.

AlBizzIA JUlibrissin, tuft flower, pinnate leaves fold down during the night, pot grown, I $-\mathrm{I} / 2 \mathrm{ft}$.

ANDROMEDA JAPONICA, green foliage, 8-i 2 in... " " variegated " "

Aralia Sieboldi, green foliage.................

" " variegated “

ARDISIA CRISPA, very fine small plant with red and white berries, pot grown

$400 \quad .3500$

$500 \quad 4500$

$200 \quad 1500$

$250 \quad 2000$

$350 \quad 3000$

" SiEBoldi, green foliage, pot grown........

“

$$
\text { variegated }
$$

،

.........

ASPIDISTRA LURIDA, variegated..................

AsTILBE JAPONICA, white flowers, large clump...

Aucuba JAponica, five central, marginal and speckled variegations, also red berry

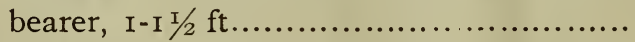

AzALEA MOLLIS, red, salmon and yellow colors

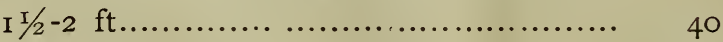

350

3000

6000

“ SchilipPENBACHI, light lilac color.

$350 \quad 3000$

“ LEDIFOLIUM, var. narcissiflorum, light purple double fiower, I ft

" LEDIFOLIUM, var. leucanthum, pure white

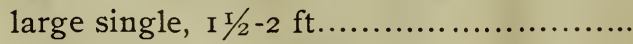

" white and pink variega

" large purple flower..........................

“ LINEARIFOLIUM, sinall narrow petals, star-like flower, white, slightly shaded with purple

$60 \quad 500$ 
Foliage, Flowering and Decorative Plants, Trees, Eitc.-Cont'd.

Each. Per io. Per roo.

Azalea Indicun, salmon red, single, white at

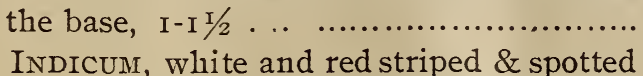

“ INDICUM, white and red striped \& spotted fuse bloomer, I ft.

Besides many other varieties.

ВАмвоо, common giant.

" fancy varieties..........................

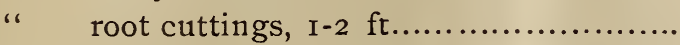

BERBERIS JAPONICA, spiny-leafed evergreen, II $\mathrm{I} / 2 \mathrm{ft}$.

“ FoRTUNI, narrow-leafed evergreen, II $\mathrm{I} / 2 \mathrm{ft}$....

BROUSSONETIA PAPYRIFERA, paper mulberry,

Seedlings I-2 ft

$50 \quad 400$

$50 \quad 400$

35300

$40 \quad 350$

$50 \quad 450$

75

500

$35 \quad 300$

$40 \quad 350$

CAMELIIA JAPONICA, single and double pink, red, variegated, etc., I 8-24 in ................ “ JAPONICA, red, variegated, etc., 24-30 in.. " " white and best variegated, I8-24 in. " " " " " $" 24-30 \mathrm{in.}$ " " white, pink, red and " $30 \cdot 36$ in. “ SASANQUA, 5 varieties, I-2 ft...............

" ThEafera, tea plant seedling...... ......

Cédrela Chinensis, beautiful foliage

Chimonanthas FRAGRANS, yellow flowers, I-I I $1 / 2$ ft.

Chrysanthemuls, best collection of large clumps with buds

Chrysanthenums, best collection of young shoots, ready in April.

Chrysantheimums, newest variety, with buds... young shoots,

ready in April.................................
Cinnamonum Camphora, seedlings, 6-I2 in......

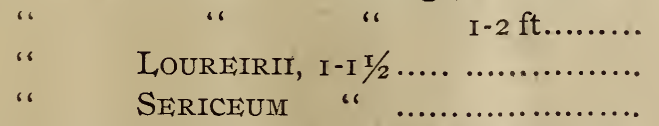

CLERODENDRON SQUAMATUM, broad leaves with red flowers, I-I $1 / 2$.

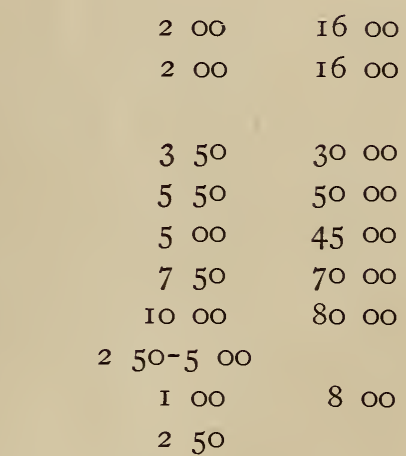

50

400

350

3200

200

I7 00

I 25 IO $\mathrm{OO}$

I $00 \quad 750$

I $\mathrm{OO}$

800

25

I 75

I 500
35300

25200

50 300 
Foliage, Flowering and Decorative Plants, Trees, Etc.-Cont'd.

Cornus MACROPHYLIA, fine foliage with white flower, $\mathrm{I}-2 \mathrm{ft}$.

Each.

Per 10.

Per $100^{\circ}$

“ MACROPHYLLA, white var. foliage, I-2 ft

" " light pink " "

CORYLUS HeTEROPHYLLA, hazel-nut, I-I I $/ 2 \mathrm{ft}$...

Crataegus Cuneata, fine foliage with red ber-

ries, $\mathrm{I}-\mathrm{I} \mathrm{I} / 2 \mathrm{ft}$

$\begin{array}{llllll}35 & 3 & 00 & 25 & 00 \\ 40 & 3 & 50 & & \\ 40 & 3 & 50 & \\ 40 & 3 & 50 & \\ 30 & 2 & 75\end{array}$

Cryptomeria JAPONICA, timber tree and also hedge plant, $2 \frac{1}{2} \mathrm{ft}$., open ground.........

CRYPTOMERIA Japonica, pot grown, $2-3 \mathrm{ft} . . . . .$.

$\begin{array}{lll}\text { " } & \text { variegated foliage.......... } \\ \text { " } & \text { " (Jindai) thick " } & \mathrm{I}-\mathrm{I} \mathrm{I} / 2 \mathrm{ft} \\ & & \text { (Bandai), dwarf, suitable }\end{array}$

lawn plant

" (Bandai), dwarf, suitable

Cryptomeria JAponica, (Koyo), dark red

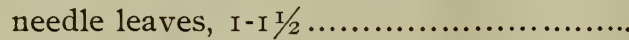

Cryptomeria JAPONICA, (Aya), whitish green foliage, I ft.................................

CRYPTOMERIA JAPONICA,(Sekka), twined, cock's comb shaped, 8-12 in.......................

Cryptomeria Japonica, (Kusari), drooping, cord-like foliage.

Cryptomeria Japonica, (Kujaku), slender cordleaved, $\mathrm{I} \mathrm{ft}$

CyPripedium Japonicum, strong pendulous flower, pot grown

60

35300

I $00 \quad 800$

250

$2 \mathrm{OO}$

200

300

I $\mathrm{OO}$

750

Cypripedium MACRANTHUM, large flower resembling calceolaria

DAPHNE ODORA, pink and white, 8 - 2 in.........

"، " "

" " " " pot grown

" GENkWA, blue flower, 6-I2 in.

DAPHNIPHYLLUMS GLAUCESCENS, thicker leaves " " $\quad$ variegated leaves

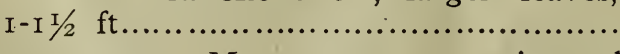

$250 \quad 2000$ I 5 I 25

$25 \quad 2 \quad 25$

$40 \quad 350$

252 O०

353 ००

$50 \quad 450$

$25 \quad 200$

DAPHNIPHYLLUMS MACROPODUM, variegated

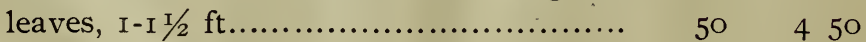

DIELYTRA SPECTABILIS...........................

DIERVILIA HORTENSIS, pink blossoms, very striking, I $\mathrm{ft}$ 
Foliage, Flowering and Decorative Plants, 'Trees, Etc.-Cont'd.

EDGEIVORTHIA PAPYRIFERA, three-fork paper mulberry, seedlings, I-2 $\mathrm{ft}$....

Each. Per Io. Per

ElAECOCCA CORDATA, wide-spreading crowntrees.

Eleagnus Pungens, red edible berry bearer, easy growth, ripening in summer, $I-I / 2 \mathrm{ft}$

“ UMBELLATA, same above,

GARDENIA RADicans, small leaved Jasmine, pot grown

“ RADICANS, double flower, pot grown

" " " " var. foliage

“ FLORIDA, pot grown, I2-I 8 in.........

Ginkgo BilodA, (or Salisburia Japonica) potgrown, I-2 ft.

Ginkgo BilodA, (or Salisburia Japonica) open

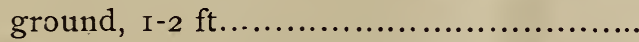

Ginkgo BILodA, (or Salisburia Japonica), variegated leaves, $\mathrm{I}-\mathrm{I} / 2 \mathrm{ft}$

HEMAMELIS JAPONICA, red flowering tree, I-2 ft.

Hydrangeas, five varieties, large clumps........ " " rooted cuttings .....

ILFX AQUIFOLIUM (Holly), I-I I $/ 2$ ft..............

“ variegated foliage, $\mathrm{I}-2 \mathrm{ft}$........

“ INTEGRA, bird-lime prepared from the bark

“ LATIFOLIA, rery fine leaves, I-2 ft...........

، “ variegated, I-2 ft................

“ Steboldi, red berries, $\mathrm{I}-2 \mathrm{ft} . \ldots \ldots \ldots . . . . . . .$.

“ " white berries, I-2 ft...............

IRIS LAEVIGATA, var. Kaempferi, IOO varieties

large and strong clumps.

IXORA STRICTA, red clustered flower.

$200 \quad 1700$

200 I5 OO

$25 \quad 2$ OO I5 OO

$25200 \quad$ I 500

20 I 50

$30 \quad 200$

$40 \quad 300$

$40 \quad 350$

$200 \quad$ I 500

I $00 \quad 800$

$40 \quad 350 \quad 3000$

$350 \quad 3000$

$40 \quad 300$

I $\mathrm{OO} \quad 8$ OO

35. 3 OO

454 o०

353 ००

25200

I $00 \quad 8$ o०

$50 \quad 400$

$50 \quad 400$

JUNIPERUS, green leaves, 2 varieties, I-I $\mathrm{x} / 2 \mathrm{ft} \ldots .$.

" white and yellow var., 2 var., $\mathrm{I}-\mathrm{I} \mathrm{I} / 2 \mathrm{ft}$

" " leaves, “ I $\mathrm{ft} . . .$. .

LYCOPODIUM PHLEGMARIA, cord-like foliage......

“ SIEBoldi, close ally of the preceding

“ JAPONICA, dark green stuff like lycopod

MAGNolias COMPRESSA, I- $\mathrm{I} / 2 \mathrm{ft}$

I 50

I 250

756 o०

353 O०

353 ००

$25 \quad 2$ ००

$50 \quad 400$

$40 \quad 350$

$40 \quad 350$

400

3500

" Salicifolia, I-2 ft.

400

35 o० 
Foliage, Flowering and Decorative Plants, Treis, Etc.-Cont' $d$.

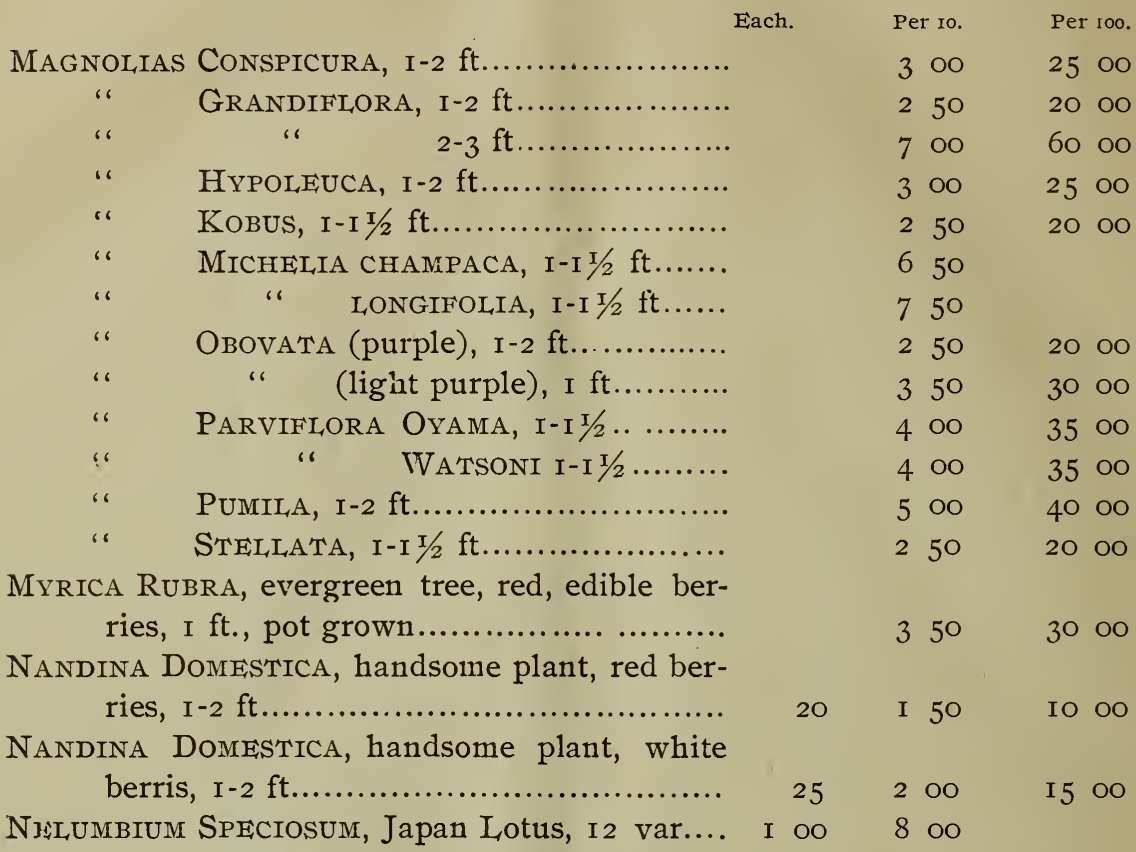

Nuphar JAPONICUM, large leaves standing above on water in late summer

OLEA FRAGRANS, very sweet yellow flowers, pot grown, I2-I 8 in

“ FRAGRANs, very sweet yellow flowers, pot grown, I8-24 in

“ FRAGRANS, very sweet yellow flowers, pot grown, 24.36 in

" FraGrans, very sweet yellow flowers, everlasting bloomers, $24-36$ in.

“ AQUIFOLIUM, strong spiny evergreens, pot grown, I-I I $/ 2$

"A AOUIFolum, var foliage pot grown

PAEONIAS, herbacious peonies, 30 varieties........

" tree peonies, roo varieties................

PARDANTHUS CHINENSIS, Blackberry Lily........

PATRINIAS SCABIOSAEFOLIA, yellow, lovely

flower, pot grown..........................

PATRINIAS VILLOSA, white, lovely flower

$30 \quad 250$

50400

$350 \quad 3000$

$500 \quad 4500$

$200 \quad 1600$

Photinia Glabra, beautiful hedge plant, $2-3 \mathrm{ft}$

300

300

$25 \quad 200$

I5 00 
Foliage, Flowering and Decorative Plants, Trees, Etc.-Cont'd.

Photinia Glabra, beautiful hedge plant, var.

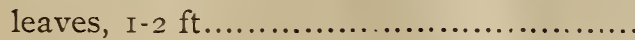

PINUS LARIX LEPTOLEPIS, deciduous pine, I-2 ft

" DEXSIFLORE, I-2 ft......

“ KORAIENSIS, “

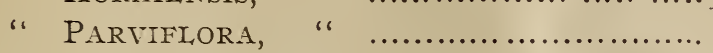

"THUNBERGII,

" dyarfed, ornamental plants, I-5 ft...........

PODOCARPUS MACROPHYLLA, open ground, I-2 ft " " $\quad$ pot grown, I-I I $/ 2 \mathrm{ft} \ldots$ " " " white var., I-2 ft...... " " " $\quad$ yellow var., I-I $1 / 2 \mathrm{ft}$. " " $\quad$ sandy spotted, I-2 ft. "، incurved foliage I$\mathrm{I} / 2 \mathrm{ft}$ Each. Per Io. Per Ioo.

$\begin{array}{cccc}40 & 3 & 50 & \\ 1 & 00 & 800 \\ 3 & 50 & 3000 \\ 4 & 00 & 3500 \\ 3 & 50 & 3000 \\ 3 & 00 & 2500\end{array}$
$50 \mathrm{cts}$. to $\$ 5$ oo each
I $O 0$
800

300

600

I 25 IO 00

70650

I $00 \quad 800$

Podocarpus Nageia, green leaves, I-2 ft........ ، ،

PRUNUS PSEUDO-CERASUS (Flowering cherry) over 20 varieties, 2-3 feet, open ground...

PSEUdO-CERASUS, I-2 ft., pot grown...

، Mune (Flowering plum), over 20 var.,

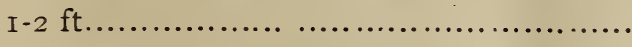

MUME (Flowering plum), rare var., I-

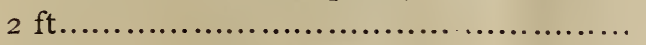

PERSICA (Flowering peach), 5 var., I-

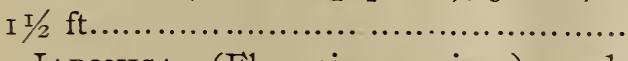

Pyrus JAPONICA, (Flowering quince), red, white and variegated.

"SPECTABILIS, profuse bloomer in May, 2$2 \mathrm{I} / 2 \mathrm{ft}$.

RAPHIOLEPIS JAPONICA, fragrant evergreen shrub $\mathrm{I}-2 \mathrm{ft}$

ROGERIA GRATISSIMA, very fragrant, white and

$\begin{array}{rrr} & 3 \text { OO } & \\ \text { I } 00 & 8 \text { oo } & \\ \text { I Oo } & 8 \text { oo } & \\ \text { Per ro. } & \text { Per Ioo. }\end{array}$

$200 \quad$ I $500 \quad$ I 2500

$300 \quad 2500$

$300 \quad 2500$

$600 \quad 5000$

$450 \quad 4000$

$250 \quad 2000$

$300 \quad 2500$

$\begin{array}{rr}250 & 20 \text { oo } \\ \text { Each. } & \text { Per 1o. }\end{array}$ purple, $I-I / 2$ ft........................\$

$75 \$ 6$ oo $\$$

RosA LAEvigATA, white and maroon, single flowers, climbing rose, I-2 ft.

$40 \quad 300$

“ Microphylia, ball-like, double flowers, i ft

$30 \quad 250$

" BANKSIAE, white flower, free bloomer, I ft

$40 \quad 350$


Foliage, Flowering and Decorative Plants, Trees, ETc.-Cont'd.

Rosa Rugosa, single rose.

white flower.

.

SERISSA JAPONICA, very beautiful

Cuspidata, I $\mathrm{T} / 2-2 \mathrm{ft}$

“

DENTATA

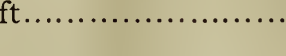

ScIAdopitys Verticillata, UMbRelita Pine,

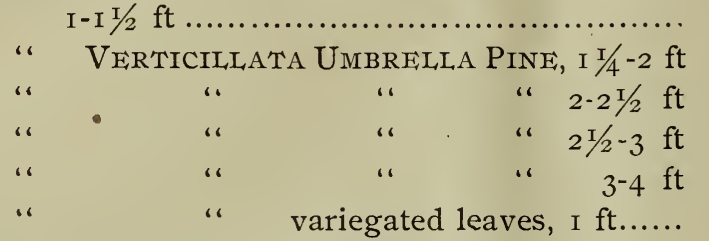

Skimmia Japonica, green foliage, pot grown,

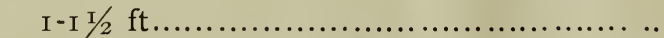

“ JAPONICA, var. foliage, pot grown, I-I $1 / 2$ $\mathrm{ft}$......

Per ro.

Per 100.

$30 \quad 250$

$30 \quad 250$

200

I 500

250

$40 \quad 350$

3000

75600

55 oo

75 I 4 OO

I 3000

$325 \quad 2700$

$450 \quad 4000$

I OO 750

$30 \quad 250$

3 oo

$75 \quad 700$

250

SPIRAEA Cantoniensis, white clustered flower..

" ARuncus, white and pink flower in cluster

STYRAX JAPONICUM, I-2 $\mathrm{ft}$

“ Obassia, 2-3 ft., very beautiful.

“ Pterostyrax Corymbosum, i-2 ft

TAMARIX CHINENSIS, pink clustered flower, I$\mathrm{I} / 2 \mathrm{ft}$

TERNSTROEMIA JAPONICA, green foliage, pot grown, I-I I/2 ft..........................

TERNSTROEMIA JAPONICA, red and white variegated, pot grawn, I-I $\mathrm{I} / 2 \mathrm{ft}$

50

2000

250

2000

250

2000

3 oo

2500

300

2500

$25 \quad 200$

$50 \quad 350$

Thuya DolABRATA, pot grown, green foliage, $I-I / 2 \mathrm{ft}$.

“ DOLABRATA, var. foliage, potgreen, I-I I/2 $\mathrm{ft}$.....

$35 \quad 300$

$40 \quad 350$

“ Dolabrata, finer foliage, pot grown, II $\mathrm{I} / 2 \mathrm{ft}$.

$40 \quad 350$

“ ОвTUSA (Hinoki) pot grown

$\begin{array}{llll}2 & 50 & 22 & 50 \\ 2 & 50 & 22 & 50 \\ 3 & 50 & & 30\end{array}$

finest leaves in regular rows...............

“ OBTUSA (or Retinosporas) pot grown, golden var. leaves

500

4000 
Foliage, Flowering and Decorative Plants, Trees, Eitc.-Cont'd.

Each. Per io. Per roo.

ThuYA OBTusa (or Retinosporas), pot grown, sil-

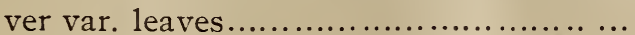

" OвTusa (or Retinosporas), beautiful dwarfed trees, I-4 ft.......................

" ОвтUSA (Kujaku), thick, bush foliage, pot grown....

“ ОвтUSA (Hotaru), splashed, white foliage, I $\mathrm{ft}$

" ORIENTALIS, folded, plain leaves, $\mathrm{I}-\mathrm{I} / 2 \mathrm{ft}$

" PISIFERA, I-I I/2 ft........................

"SQUARROSA, I-I I $1 / 2 \mathrm{ft}$

VIBURNUM ODORATISSIMUM, green foliage, with red berries

" ODORATISSIMUM, variegated foliage.......

“ Plicatum, Japan Snowball, beautiful foliage

“ Plicatum, Japan Snowball, variegated...

ZANTHOXYLUM PIPERITUM seedlings..............

Zoysia Pungens, Japanese grass for edging, turf ground, etc., per roo lbs

$650 \quad 6000$ I 00 to 5000

$\begin{array}{llllll} & 3 & 00 & & 27 & 50 \\ & 3 & 00 & & 27 & 50 \\ 2 & 50 & & 22 & 50 \\ & 2 & 00 & & & \\ & 2 & 00 & & & \\ & & & & & \\ 35 & 3 & 00 & & & \\ 40 & 3 & 50 & & & \\ & & & & & \\ & 3 & 50 & & 30 & 00 \\ & 5 & 00 & & 45 & 00 \\ 2 & 50 & & \end{array}$

IO 00

\section{Climbers and Creepers.}

Actinidia Polygana, white flower in summer, deciduous climber.

Each. Per Io.

Per roo.

AkeBIA QUINATA, hardy ornamental foliage climber, yielding edible fruit

$\$ 50 \$ 400$

" LoBATA, close ally of the preceding ex-

cept 3 leaves on one stalk..................
BERCHEMIA RACEMOSA, flexible woudy vine, very strong.................................

"RACEMOSA, variegated leaves.

Ceinastrus ARbiculata, highly ornamental woody vine leaving small yellow fruit after the leaves fall off

CLEMATIS FLORIDA, greenish white flower. double white flower, tipped $30 \quad 250$

$50 \quad 400$

$30 \quad 250$

50 $\quad 400$

with green near the center. 
Climbers AND CREEPERS-Continued.

Clematis Paniculata, hardy climber, with fragrant white double flowers in cluster......

“ PATENS, violet, large petal, highly recommended flower.

" PATENS, double, fine white flower... ..... HETEROSMILAX JAPONICA, ornamental climber.. Hoya CARnosa, thick succulent leaved climber, pink waxy flower....

" CARNOSA, central variegated................

" " marginal variegated..............

Hydrangea Petiolaris, woody vine, white umbel flowers

IPOMAA HEDERACEA, Japanese morning glory.. KADSURA JAPONICA, woody climber, with red berries.

" JAPONICA, woody climber, with white berries.

“ JAPONICA, woody climber, with variegated leaves.

LYCODIUM JAPONICUM, climbing fern..............

MILLETIA JAPONICA, small climber, wisterialike flower................................

Puellyaria Thunbergiana, shading climber....

Rubus Rosifolius, var. Coronarius, white, double climbing rose......................

Stauntonta Hexaphylia, white flower, edible fruit.

TECOMA GRANDIFLORA, bell shaped, orange flower.

TRACHELOSPERMUM JASMINOIDES, small leaved fine climber.

Wisteria Chinensis, blue flower, 3-5 ft. ........ double blue flower ...........

BRACHYBOTRYA, white flower, $3-5 \mathrm{ft}$..... double white flower...

\begin{tabular}{|c|c|}
\hline Each. & Per 10. \\
\hline 35 & 300 \\
\hline 60 & 500 \\
\hline 60 & 500 \\
\hline 30 & 250 \\
\hline 35 & 300 \\
\hline $5^{\circ}$ & 400 \\
\hline 60 & $5 \circ 0$ \\
\hline 40 & 350 \\
\hline IO & 75 \\
\hline 35 & 300 \\
\hline 35 & 300 \\
\hline 50 & 400 \\
\hline 25 & 200 \\
\hline 40 & 350 \\
\hline 40 & 350 \\
\hline 30 & 250 \\
\hline 50 & 350 \\
\hline 40 & 350 \\
\hline 40 & 360 \\
\hline 40 & 350 \\
\hline $\mathrm{OO}$ & 7.50 \\
\hline 40 & 350 \\
\hline $\mathrm{OO}$ & 750 \\
\hline
\end{tabular}

Per Ioo.

oo

00

00

Do

OO

50

75

300

300

400

50

50

50

50

360

350

7.50

750

\section{Ferns.}

Large clumps in 4-6 in. pot.

Adiantum Monochlamys, hairy soft fronds, Each. Per ro. Per 100. black stems, large clumps................... 5० 4 ०० 
FERNS-Continued.

Angiopteris EUEcta, large leaves come out from clumy trunk........................... Aspidium CORDIFOLIUM, long frond hanging 2-3 ft. long.

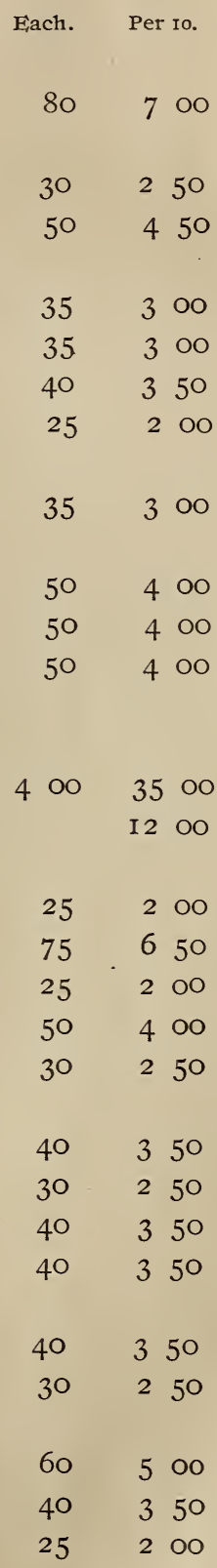

Per Ioo.

“ LEPIDOCAULON, dark green creeping fern

“ FALCATUM, fleshy trunks like cycas, revolutta's leaves

“ FELIX-MAS, thick blade leaves.

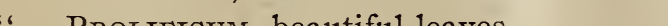

" Prolificum, beautiful leaves...............

“ Tripteron, cross frond fern..... ............

“ VIRIDESCENS, both sides of foliage are equally showing.

Asplennium Nidus, large broad blade reaching to

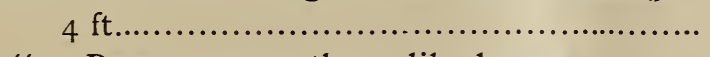

RUTAFOLIUM, thuya-like leaves. ...........

Cyathea Spinulosa, Japanese tree fern, $1 / 2 \mathrm{ft} . .$. many artificial model works are made by bending roots, ball, ring, half-moon, etc.

" BullatA, house, ship, etc.

" tenuifolia, wire-like stems

with yellowish green frond..................

LOMARIA NIPPONICA, richly curled, comb-shaped

LyGodiUM JAPONICUM, climbing fern.................

Meniscium Simplex, curious broad blades........

ONOClEA SENSIbILIs, leaves fall during Winter..

Osmanda Claytoniana, foliage appears in

A pril with yellow flower...................

“ REGALIS, very bushy

Polypodium Lingua, broad and thick blade....

PTERIS CRETICA, white unclear stripe.............

"SERRULATA, thread-like long leaves (not serrated)

“ SERRULATA, broader leaves

PSILOTUM TRIQUESTRUM, spike flower, very fine plant.

SCOLOPENDRIUM VULGARE, small broad blade...

WOODWARDIA ORIENTALIS.

$25 \quad 200$ 


\section{Orchids.}

AERIDES JAPONICA, white with purple spots,

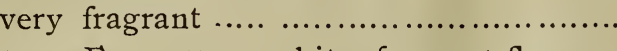

ANGLACUM FALCATUM, white fragrant flowers, attached to old wood, 8-12 in.................

“. FALCATUM, pot grown...................

" " foliis albo-marginatis...

Bletia Hyacinthina, variegated leaves, 4-5 shoots in each pot.

“ Hyacinthina, white flower, 4-5 shoots in each pot

“ Hyacinthina, purple flower, 4-5 shoots in each pot.......................................

CyPRIPIDIUM DeBILE, small pretty orchid......... “ JAPONICA, strong, pendulons flower on stalk between two large round leaves....

Cypripidium Macranthum, large flower, resembling calceolaria

Calanthe Discolor, pink with erecting spike

(6)

6 yellow canary yellow, largest flower

6 JAPONICA, pink and white

" TEXTORI, pale yellow flower

Cymbidium Ensifolium, very fragrant, greenish white flower

Cymbidium Virens, greenish white flower, with purple spots

DENDROBIUM MONILIFORME, light pink, 3 in mass 6

$$
\text { " yellow, }
$$

"

EPIPACTIS GiganTEA

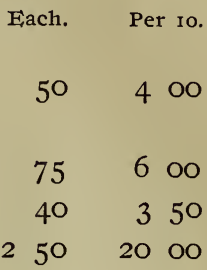

$40 \quad 350$

30

250

252200

$30 \quad 250$

20

I 50

30

250

$30 \quad 250$

$75 \quad 500$

I 50 IO 00

$50 \quad 450$

$40 \quad 350$

200

$40 \quad 350$

$60 \quad 500$

Goodyera Velutina, reddish purple, lustrous leaves...................................

HAVENARIA RADIATA, variegaten leaves..........

HAVENARIA RADIATA, white wing-like flower, yields tuber root...........................

KANRAN, fragrant greenish lilac flowers, $5^{-6}$

shoots each................................
LINSIA TERES, peculiar stick-like stem with brown and white flowers

25200

I 00

I5 I OO

IO

50

I 00 
ORCHIDS-Continued.

Phajus Grandiflorus, lipped flower, broad lustrous foliage................................

“ Maculatus, yellow flower................

" " " " yellow spots on leaves........................................

Soshin, pure white flowers with long leaves, very fragrant, $5-6$ shoots each................. I 25

“ white marginal leaves, 5.6 shoots each..... I 75

\section{Palms.}

Chamaerops Exceisa, rapid growth, easy culture, 8-I2 in

Each. Fer Io. Per 100

Chamaerops, dwarf variety, attaining io ft., hard and smaller leaves, in 4 in. pot.......

Chamaerops, dwarf variety, in 7 in. pot......... “"
" 3 years old
3 years old.............

Cycas Revoluta, (Sago Palm), with fronds, according to foliage and size of trunks, ......

“ Revoluta, (Sago Palın), fronds cut off. \$7 per roo lbs., \$60 per rooo Livistonia Chinensis, handsome Chinese fan palm, I-I I $/ 2 \mathrm{ft}$

RAPHIS FLABELLIFORMIS, bamboo palm, very attractive indoor plant, 5 in. to $4 \mathrm{ft}$. (per IOOO, $\$ 320$ )

“ FlABELLIFORMis, variegated.

4 oo

$35 \circ 0$

“ KuANontsik, broader leaves of Flab. 5 in. to $2 \mathrm{~T} / 2 \mathrm{ft}$

" KUANONTSIK, variegated foliage.

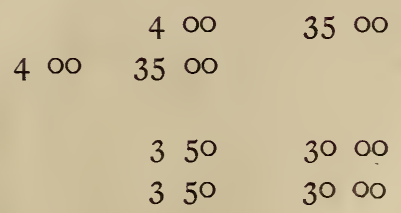

\section{Fruit Trees.}

Persimmons, best varieties, $3-4 \mathrm{ft}$

Per Io.

Per Ioo.

Per 1000.

PltM TREes, "

" $3-4 \mathrm{ft}$

$\$ 6$ oo

$\$ 55$ ○०

" Suika or Satsuma, 3 years cultivated in Oakland and bears fruit, $2-3 \mathrm{ft}$ I $\mathrm{OO}$

$800 \quad 7000$ $200 \quad$ I 500 
FrUIT TrEes-Continued.

Giant Chestnut, open ground, 3-4 ft..........

JAPAN QUINCE.

LoQUAT JAPONICA, new variety, 4-5 times larger

than common fruits, $I-I / 2 \mathrm{ft}$.

\begin{tabular}{|c|c|c|}
\hline \multirow[t]{2}{*}{ Per io } & $\begin{array}{l}\text { Per roo. } \\
8 \text { oo }\end{array}$ & $\begin{array}{l}\text { Per } 1000 \\
7000\end{array}$ \\
\hline & I3 00 & \\
\hline 400 & 35 оо & \\
\hline 350 & 3000 & \\
\hline & 550 & 5000 \\
\hline 300 & 2000 & \\
\hline 200 & $\mathrm{I}_{5} \mathrm{OO}$ & \\
\hline 80 & 600 & 40 \\
\hline I 00 & 800 & 6000 \\
\hline I 25 & IO 00 & \\
\hline
\end{tabular}

GIANT LoQUAT, I I $/ 2-2 \mathrm{ft} \ldots \ldots \ldots \ldots \ldots \ldots \ldots \ldots \ldots$

Chestnut Seedlings, for grafting................

JAPANESE FIG TREES, pot grown...

“ GRAPE Vines, I I $/ 2-2 \mathrm{ft}$

Rubus Insisus, yellow, sweet berries, open ground, I ft.

“ Rosifolius, wineberry, red, sweet berries

Juglans Sieboldiana, seedling, I-2 $1 / 2 \mathrm{ft} \ldots \ldots \ldots$.

ORANGE TREES.

UNSHIU Seedless and KII Oranges, pot grown, I 8-24 in., free from scales ..................

SAME, open ground, I8-24 in

\begin{tabular}{|c|c|c|c|}
\hline 350 & 30 & OO & \\
\hline 200 & I 7 & 50 & I 50 OO \\
\hline 250 & 22 & $\mathrm{OO}$ & 20000 \\
\hline & 2 & 50 & $20 \mathrm{OC}$ \\
\hline & 4 & OO & $35 \mathrm{OC}$ \\
\hline 50 & 35 & O० & \\
\hline OO & I 5 & ০০ & \\
\hline 50 & 30 & oo & \\
\hline 250 & 20 & $\mathrm{OO}$ & \\
\hline 50 & 35 & Oo & \\
\hline $\mathrm{oO}$ & 35 & OO & \\
\hline & 2 & 50 & 2000 \\
\hline & 2 & 50 & 2000 \\
\hline
\end{tabular}

SATSUMA or CANTON Hybrid, open ground.......

" " " pot grown.........

CiTRUS JAPONICA, pot grown, I-2 ft.............

" " open ground, without fruit, I-2 ft..................................

" JAPONICA, larger fruit, new variety, pot

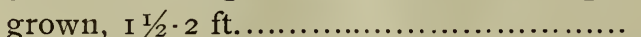

“ JAPONICA, open ground, $\mathrm{I} / 2-2 \mathrm{ft} \ldots \ldots \ldots \ldots$

“ Bigardia (Bitter Orange), pot grown, for ornamental purposes, I $1 / 2-2 \mathrm{ft} \ldots \ldots \ldots \ldots \ldots . .$.

“ DECMANA, very large thick-skinned fruit, pot grown, I $1 / 2-2 \mathrm{ft} . . \ldots \ldots \ldots \ldots \ldots \ldots$.

Citrus Trifolia, i ft.

PEAR SEEDLING, for grafting stock.

250

2000

\section{Seeds.}

Abies Alcoguiana

Per 1b.

Per $1 \mathrm{~b}$.

" Brachyphylla...............4 50

" Firma.

I 00

Abies Mariesi.

550

" Sacchalinensis.............5 50

" Polita........................ 50 


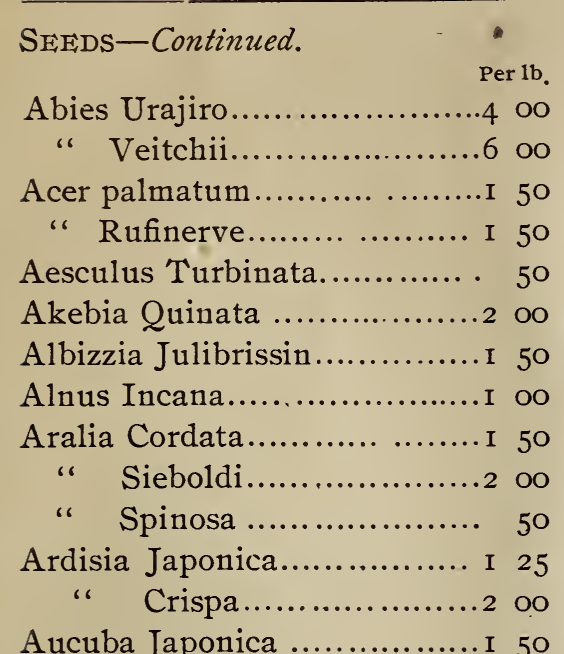

Azalea Mollis, etc., per packet 50 Broussonetia Papyrifera......... 50 Camellia Japonica............... 50 “ Theifera (Tea)........ 50

Canavallia Incurva............ 50

Castanea Vulgaris, (chestnut) \$I2 per roo $1 \mathrm{bs} . . . . \ldots \ldots \ldots \ldots . . . .25$

Celastrus Articulata............. o

Cephalotaxus Drupacea........ 50

Cercidiphyllum Japoicum.....5

Chamaerop Excelsa.............. I oo

Cinnamomum Camphora........ 50

، Loureiri..........

Cornus

Crataegus Cuneata...............

“ Sanguinea...........2 oo

Corylus Heterophylla........... 3 oо

Crinum Asiaticum............... I oo

Cryptomeria Japonica............ 50

Cycas Revoluta................. 50

Dianthus, (single pink) per

packet ....................... 50

Deutzia Scabra.................. I o०

Diosyras Kaki (Persimmon)... 50

“ Lotus ". ... I

Eleagnus Longipes............2 o०
Per lb.

Eleagnus Pungens.............. I O5 $_{5}$

Enkianthus Japonicus...........3 00

Euonymus Statua...............2 00

Euscaphis Staphyleodes......... I 50

Fagus Sylvatica................ 75

Gardenia Florida...............2 50

Gleditschia Japonica ........... 50

Hovenia Dulcis.................2

Hammamelis Japonica...........2 50

Hypericum erectum.............. I 50

Irex Crenata....................2 o०

" Integra.....................2

“ Sieboldi..................... I 50

Iris Kaempferi..................

Juglans Cordiformis (Walnut) 35 “ Sieboldi “, 25

Juniperus Rigida...............2 o०

Lagenaria Vulgaris.............. I

Lagerstroemia Indica...........2 00

Larix Leptolepis...............4

Ligustrnm Ibota.............. I 50

“ Japonicum............

Lindera Sericea................. I 50

Luffa Petola....................2 oo

Lycium Chinensis.. ............ I 50

Magnolia Hypoleuca ........... 50

، Kobus................. I 50

Morus Alba.....................5

Myrica Rubra................... 3 oo

Nandina Domestica.............. 50

Nelumbium Speciosum (Lotus) I oO

Oryza Sativa (Rice) \$I 5 per I oo

lbs............................ 20

Paeonia Arborea................ I 50

Paulownia Impeliaris............ 3 oo

Photinia Glabra.................2 о०

“ Villosa.................. I 50

Picea Alcoquana.................5

“ Polita.......................5 00

Pinus Densiflora................ I о०

" Koraiensis................4 
SEEDS-Continued.

- Per lb.

Pinus Parviflora............... I 50

“ Thunbergii............... 75

Podocarpus Macrophylla........ oo

Prumula Japonica..............5 ००

Prunus Japonica................ o

Pterostyrax Corymbosum.....5 00

Pyrus ussuriensis (Pear) \$30

per ro 1bs., \$275 per roo 1bs.3 $5^{\circ}$

Quercus Dentata................ 50

" Cuspidata...............

“ Serrata................. 25

Raphiolepis Japonica................. о

Rhus Semi Alata.... ................. 50

" Succedanea..................... I oo

“ Vernicifera........................ ०

Rosa Rugosa.........................2 50

Rubus....................per packet 50

Salisburia Adiantifolia (or

Ginkgo Biloba).................. 50

Sambucas Recemosa............. o०

Sapindus Mukorosi............. 30

Sciadopitys Verticillata (Um-

brella Pine) .................. 3 o

Smyrax China.... ............ 75

Sophora Japonica................2 00

Styrax Japonica................2 00

" Obassia..................3 00

Th,uya Dolabrata...............4 ००

“ Obtusa.................. I 50

" Orientalis Pendula...... I 50

Torreya Nucifera............... 50

Tsuga Sieboldi..................5 00

Viburnum Dilatatum......... 2 oo

" Odoratissimum..... 30

Vitis Labrusca..................2 oo

" Pentaphylla................ 50

Wisteria, purple................ I oo

" white.................. I 25

Zanthoxyllum Piperitum....... I 00

Zelkowa Keaki..................2 50

Zizyphus Vulgaris............. $5^{\circ}$

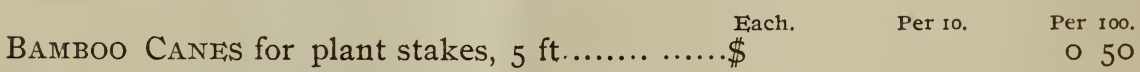

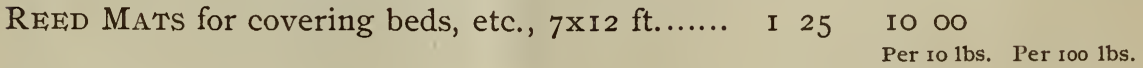

DRIED MOSs for packing, etc.................... 50 ० 50

\section{$\cong$ \\ Bulbs and Fruit Trees.}

Special rates given for orders of Io,oco or more.

Our Nursery is the most extensive one in Japan, and we will fill with promptness and reliability any order entrusted to us for any plants or trees named in this Catalogue, or for any others grown in Japan and not named in the foregoing list, at lower rates than can be obtained elsewhere.

OAKLAND, Cal.. May, i893.

P. O. Box 453 . 